\title{
Insulin and value-added therapy
}

\author{
Sanjay Kalra, Yashdeep Gupta ${ }^{1}$, Manash P Baruah ${ }^{2}$ \\ Department of Endocrinology, Bharti Hospital, Karnal, Haryana, ${ }^{1}$ Department of Medicine, Government Medical College and Hospital, \\ Chandigarh, ${ }^{2}$ Department of Endocrinology, Excel Care Hospitals, Guwahati, Assam, India
}

\section{INSULIN THERAPY}

We are all familiar with the abbreviation VAT, which stands for value-added tax. Most readers take VAT as an integral, necessary part of life and understand the benefits that accrue from it.

Insulin therapy too is acknowledged as an essential aspect of diabetes management, with unquestionable benefits. In spite of great strides in the manufacture, presentation and quality of this molecule, however, we have not achieved commensurable improvement in glycemic control. Data from across the world reports sub optimal control of persons started on insulin therapy. The best of randomized controlled trials and observational studies are unable to achieve target glycemic levels in all enrolled subjects. This happens in spite of aggressive treat to-target protocols used.

What implication does this have for clinical practice? One suggestion, expounded upon in this article, is the need for value added therapy (VAT), in addition to insulin therapy.

\section{VAT}

For insulin to be effective, the prescription must be accompanied by VAT. These value, added services are essential if one has to achieve pre-set glycemic targets, whereas minimizing adverse events such as hypoglycemia. The VAT improves patient acceptance of injectable therapy, ensures adherence and persistence with treatment and

\begin{tabular}{|l|l|}
\hline \multicolumn{2}{|c|}{ Access this article online } \\
\hline Quick Response Code: & Website: \\
\hline & www.josh.net \\
\hline & \\
\hline
\end{tabular}

encourages patient provider bonding and communication [Table 1]. VAT includes an array of items. Some are treatments in their own right, while others are ways of administering treatment. A few of the VAT can work with or without insulins and a few are intricately tied to insulin. Although some insulin-related VATs can be measured or qualified, others remain intangible [Table 2].

\section{TANGIBLE VAT}

Certain forms of VAT to insulin delivery devices may influence the acceptability and tolerability of insulin treatment. Recent data related to current state-of the- art insulin pens, such as Novo Flex, Solostar and Humapen, for example, highlights the advantages associated with their use. Modern insulin syringes, which are shorter and thinner $(4-6 \mathrm{~mm}, 29 \mathrm{G})$ also ensure a comfortable injection experience.

Appropriate use of other drugs, in combination with insulin, is an often-reviewed topic. ${ }^{[1]}$ Usage of drugs such as metformin, glucagon like peptide- 1 receptor agonists (GLP-1 RA), in appropriate clinical settings, helps reduce insulin dose requirement and allows greater chances of achieving glycemic targets. ${ }^{[2]}$

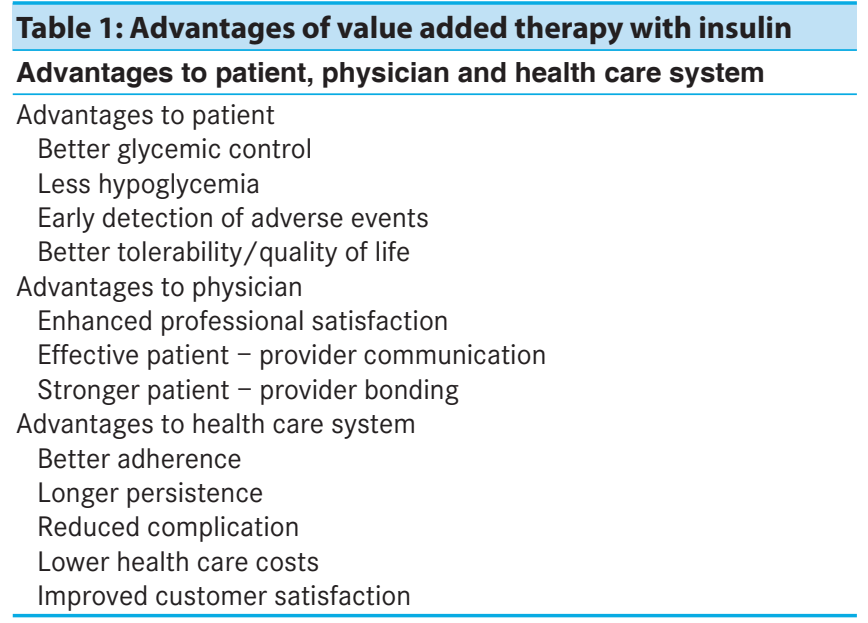

Corresponding Author: Dr. Sanjay Kalra, Department of Obstetrics and Gynaecology, Bharti Hospital, Kunjpura Road, Karnal - 132 001, Haryana, India. E-mail: brideknl@gmail.com 


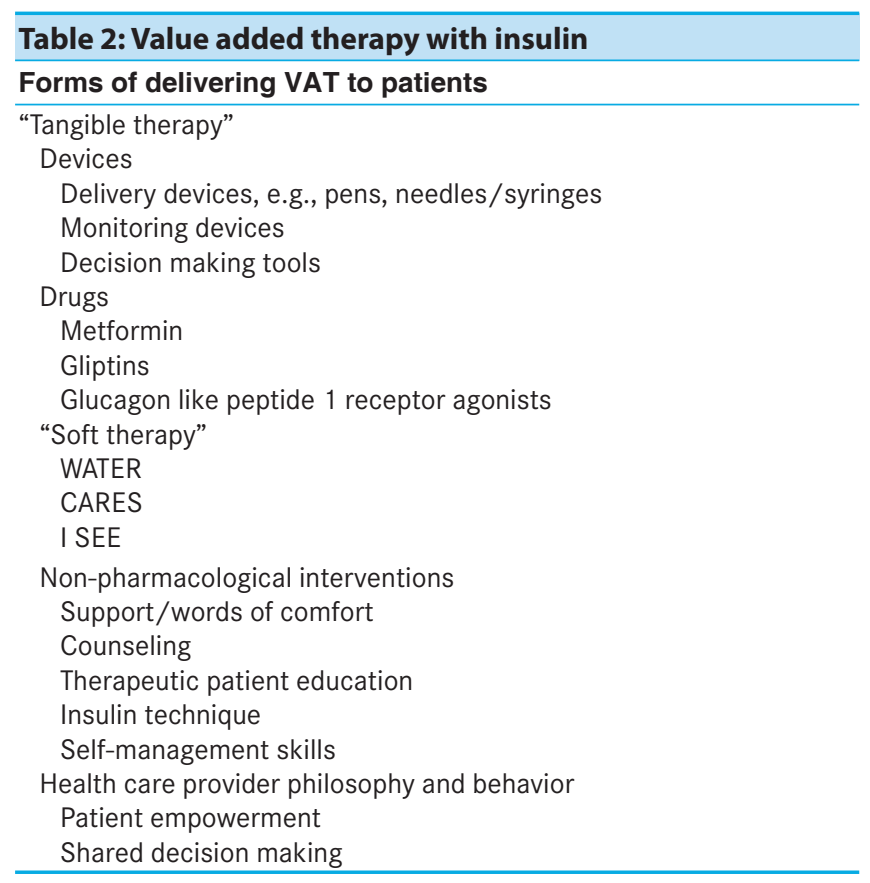

Availability of multiple insulin preparations, including ultra-long acting, ${ }^{[3]}$ biphasic premixed and rapid acting insulin analogues allows a wider range of molecules to choose from. Newer insulins such as degludec also allow flexibility in time of administration. ${ }^{[3]}$ This ability to exercise choice is perceived as empowerment and VAT by the person with diabetes.

\section{SOFt VAlue AdDED SERVICES}

"Soft" VAT describes not only the non-pharmacological interventions which should be offered with an insulin prescription, but also the ideal way in which patientprovider conversation should be conducted, as well as the underlying philosophy behind this interaction.

An insulin prescription alone is of no value. This prescription becomes meaningful and takes on a dynamic "life" of its own, if it is made response to the recipient's biological, psychological and social structure. To do so, one needs to offer continuous diabetes support, counseling and education..$^{[4]}$ All these words: Support, counseling and education, may have varying meanings for different people. Offered in tandem with each other, preferably in a structured manner; facilities and resources exist; these aspects of VAT certainly improve the efficacy, safety and tolerability of insulin.

The underlying philosophy behind diabetes care is a person-centered one. Putting this concept into practice involves him or her in shared decision making and choosing the correct insulin regime in consultation with each other. Cares water and $1 \mathrm{~s}$ are models which help diabetes care professionals develop necessary attributes or skills, use them to improve dialogue with patients and ensure adequate empowerment. ${ }^{[4]}$ Use of these concepts, along with what we term "words of comfort," enhances the value of insulin.

Specific non-pharmacological interventions have also been demonstrated to act as value additions with insulin therapy. These include diabetes support, counseling, therapeutic patient education and insulin technique demonstration. All these interventions are evidence backed VAT and must be administered as co-therapy with insulin. The aim is to optimize self-management skills and hence that insulin treatment can be utilized to achieve desired outcomes.

\section{A Holistic Perspective}

We have excellent insulin preparations and delivery devices available to us today. Modern insulin analogues, including the ultra-long acting degludec and various rapid acting molecules, provide physiological replacement for basal and prandial requirements. Premixed biphasic mixtures of aspart and lispro offer multiple ratios of both basal and prandial insulin, designed to improve patient adherence and convenience.

Yet, we are far from achieving adequate glycemic control for the majority of our patients with diabetes.

What is needed to achieve this goal? The addition of VAT described above, in a universal, yet person-centered, person responsive manner, may help in this aim.

To do so, we need to work simultaneously on various fronts. The concepts enumerated in this communication must be made part of diabetology curricula at all levels, ranging from undergraduate to fellowship, to continuing medical education programs. Patient involvement in diabetes therapy must be encouraged, at a micro-level (individual, clinic-based) as well as a macro-level (public awareness, advocacy). "Professional skills in personal motivation" is the term used by Phillip Home (1997) to describe this.

VAT is a shared responsibility, involving bidirectional communication. The person with diabetes, too, must shoulder the burden of making VAT work. "Means of motivation or self-discipline" must be found and coupled with education and the ability to make "consistent use of the information" obtained from self-monitoring data. This will help realize the potential benefits of modern insulin therapy, while avoiding hypoglycemia and other adverse events. 
Manufactures of pharmaceutical products continue to assist in this process by developing diagnostic and monitoring devices, as well as safe, effective insulins and accurate, patient-friendly delivery devices. Concerted teamwork by all stakeholders, including diabetes are professionals and persons with diabetes, should help us utilize VAT, as an adjunct to insulin and experience the full range of benefits that it has to offer.

\section{REFERENCES}

1. Nadeau DA. Physiologic and weight-focused treatment strategies for managing type 2 diabetes mellitus: The metformin, glucagon- like peptide-1 receptor agonist, and insulin (MGI) approach Postgrad Med 2013;125:112-26.

2. Cohen ND, Audehm R, Pretorius E, Kaye J, Chapman LH, Colagiuri $\mathrm{S}$. The rationale for combining GLP-1 receptor agonists with basal insulin. Med J Aust 2013;199:246-9.

3. Kalra S, Unnikrishnan AG, Baruah M, Kalra B. Degludec insulin: A novel basal insulin. Indian $\mathrm{J}$ Endocrinol Metab 2011;15:S12-6.

4. Kalra S, Kalra B, Batra P. Patient motivation for insulin/injectable therapy: The Karnal model. Int J Clin Cases Invest 2010;1:11-5.

How to cite this article: Kalra S, Gupta Y, Baruah MP. Insulin and value-added therapy. J Soc Health Diabetes 2014;2:59-61.

Source of Support: Nil. Conflict of Interest: None declared. 